\title{
Effects of vasodilator treatment with felodipine on haemodynamic responses to treadmill exercise in congestive heart failure
}

\author{
A D TIMMIS, P SMYTH, J F KENNY, S CAMPBELL, D E JEWITT \\ From the Department of Cardiology, King's College Hospital, London
}

SUMMARY Treatment with vasodilators in heart failure has not always produced a useful improvement in the haemodynamic responses to exercise, and in many cases early drug tolerance has further limited the potential of this type of treatment. In a study to evaluate the efficacy of felodipine, a new calcium antagonist with selective vasodilator properties, in the management of congestive heart failure 10 patients with congestive heart failure underwent treadmill exercise testing before and during oral treatment with felodipine $30 \mathrm{mg}$ daily. At every level of exercise felodipine lowered the pulmonary capillary wedge pressure, whereas cardiac index and stroke index increased considerably. The haemodynamic improvement was associated with an increase in the duration of exercise to exhaustion. Importantly, these beneficial effects were sustained throughout four weeks of treatment without evidence of drug tolerance. These observations suggest a useful role for felodipine in the long term management of congestive heart failure.

In patients with chronic congestive heart failure exercise related fatigue and dyspnoea are the commonest and most troublesome symptoms. The reduced capacity of the failing heart to increase output to meet the metabolic demands of working muscle and the exagerated rise in left atrial pressure largely account for these symptoms. A critical evaluation of specific antifailure treatment must, therefore, examine the influence it has on the haemodynamic responses to exercise. An additional consideration is the long term efficacy of treatment. This is more difficult to assess since the natural history of congestive heart failure involves a variably progressive deterioration in left ventricular function which is not easily distinguished from the development of drug tolerance.

Despite the widespread use of vasodilator agents in congestive heart failure considerable uncertainty still exists concerning the effects of these agents on the haemodynamic responses to exercise and on maximal exercise capacity. ${ }^{1}$ Moreover, the long term efficacy of vasodilators has been questioned in several reports. ${ }^{2-4}$ In the present study we examined these

Requests for reprints to Dr A D Timmis, Department of Cardiology, King's College Hospital, Denmark Hill, London SE5 9RS.

Accepted for publication 15 May 1984 issues using felodipine, a new calcium antagonist with selective vasodilator activity. ${ }^{56}$ Calcium antagonists have not previously been evaluated in the outpatient management of congestive heart failure. Beneficial haemodynamic and metabolic responses have, however, been shown when felodipine is given acutely to patients with severe heart failure. ${ }^{78} \mathrm{We}$ report the effects of felodipine on the haemodynamic responses to treadmill exercise during long term treatment.

\section{Patients and methods}

\section{PATIENT SELECTION}

Ten patients (nine men and one woman, age range 38-66 years) were studied (Table 1). All were selected on the basis of moderate to severe congestive heart failure which was symptomatic (New York Heart Association functional class II and III) despite treatment with diuretics. Five were receiving digoxin. Cardiac catheterisation performed within 12 months of the study had confirmed impaired left ventricular function in every case with a mean (SEM) angiographic ejection fraction of only $23(2) \%$. Five of the group had three vessel coronary artery disease and the remainder congestive cardiomyopathy of unknown aetiology. The patients were all in stable sinus rhythm. Written informed consent was obtained from 
Table 1 Characteristics of 10 patients with congestive heart failure

\begin{tabular}{|c|c|c|c|c|c|}
\hline Case No & $\begin{array}{l}\text { Age (yr) } \\
\text { and sex }\end{array}$ & Diagnosis & NYHA class & $\begin{array}{l}\text { Angiographic } \\
E F(\%)\end{array}$ & $\begin{array}{l}\text { Daily frusemide } \\
\text { dosage (mg) }\end{array}$ \\
\hline $\begin{array}{r}1 \\
2 \\
3 \\
4 \\
5 \\
6 \\
7 \\
8 \\
9 \\
10\end{array}$ & $\begin{array}{l}53 M \\
53 M \\
63 M \\
38 M \\
56 F \\
66 M \\
45 M \\
43 M \\
56 M \\
55 M\end{array}$ & $\begin{array}{l}\text { CAD } \\
\text { CAD } \\
\text { CAD } \\
\text { CAD } \\
\text { CCM } \\
\text { CAD } \\
\text { CCM } \\
\text { CCM } \\
\text { CCM } \\
\text { CCM }\end{array}$ & $\begin{array}{l}\text { III } \\
\text { III } \\
\text { II } \\
\text { III } \\
\text { III } \\
\text { II } \\
\text { II } \\
\text { II } \\
\text { III } \\
\text { II }\end{array}$ & $\begin{array}{l}13 \\
27 \\
33 \\
10 \\
23 \\
29 \\
26 \\
24 \\
19 \\
23\end{array}$ & $\begin{array}{c}40 \\
500 \\
40^{\star} \\
240 \\
80 \\
80^{\star} \\
250 \\
80 \\
250 \\
40\end{array}$ \\
\hline
\end{tabular}

*During felodipine treatment the diuretic dose was halved in these patients because of postural hypotension related to excessive pooling of blood.

$\mathrm{CAD}$, coronary artery disease; CCM, congestive cardiomyopathy; EF, ejection fraction.

each patient before inclusion in the study.

\section{STUDY PROTOCOL}

All vasodilator treatment was stopped at least four days before the study. Treatment with digoxin and diuretics was maintained throughout the 30 days of the study, although in two cases a small reduction in diuretic dosage was necessary after the introduction of felodipine. Exercise studies were always performed between 1600 and $1700 \mathrm{~h}$ after a four hour fast. The morning dose of diuretics and other regular medications was not withheld. Each patient underwent a total of five exercise tests. The first, a preliminary test, permitted an assessment of maximal exercise capacity and was performed without invasive haemodynamic measurements. On day 1 of the study control haemodynamic data during exercise were obtained after which treatment with oral felodipine 10 mg three times a day was started. Further exercise tests were performed after two days and four weeks of treatment (days 3 and 28 respectively). After day 28 felodipine was withdrawn, and placebo tablets were substituted. Two days later (day 30 ) the final exercise test was performed. Plasma samples for felodipine assay were obtained immediately before exercise testing on each of the study days.

able 2 Effects of felodipine on haemodynamic responses and oxygen metabolism at rest (resting 1), at different levels of treadmill exercise, and one inute after maximal exercise (resting 2). Values are mean (SEM)

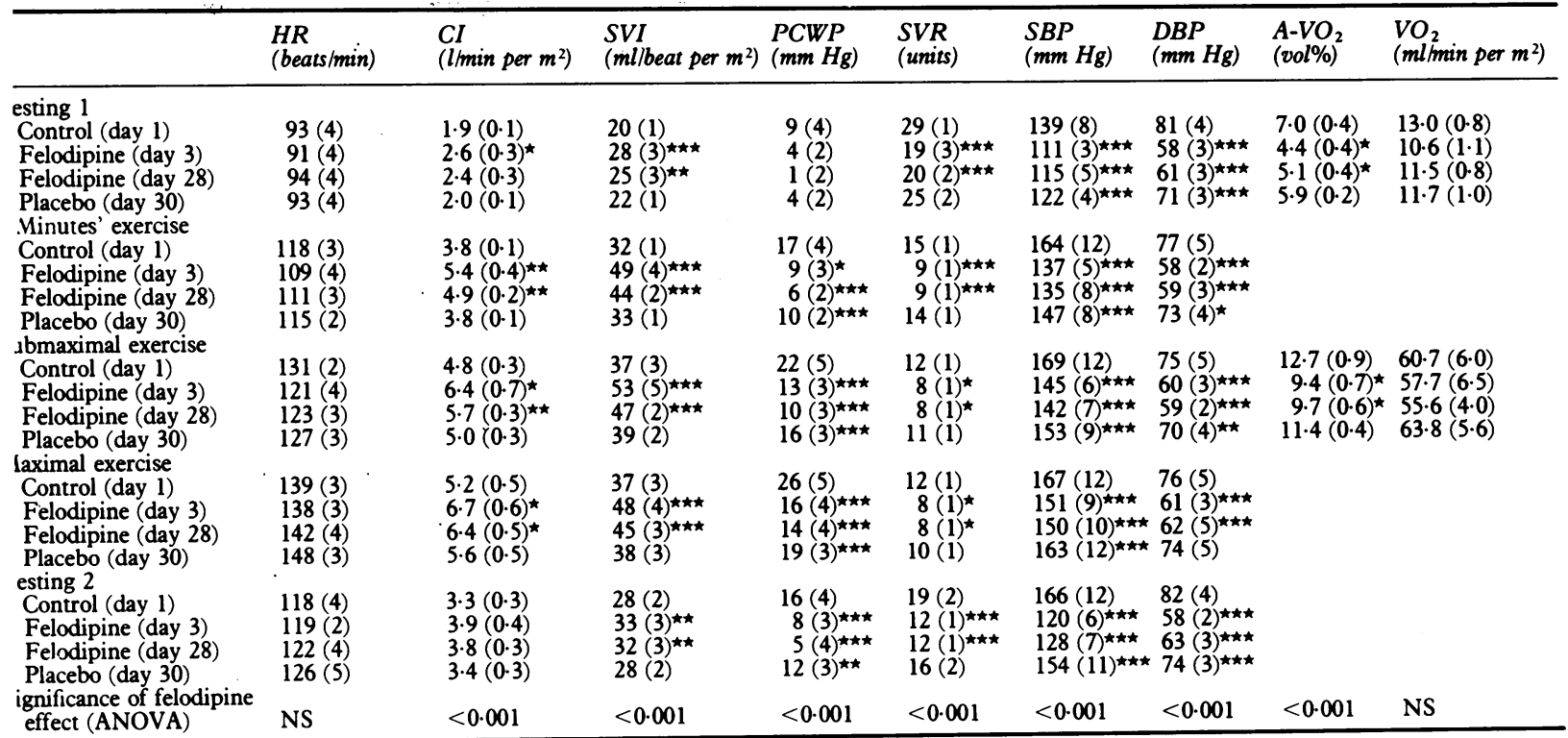

ignificance (p) of individual changes with respect to control: $\star<0.05, \star \star<0.01, \star \star \star<0.001$.

R, heart rate; CI, cardiac index; SVI, stroke volume index; PCWP, pulmonary capillary wedge pressure; SVR, systemic vascular resistance; SBP; systolic lood pressure; DBP, diastolic blood pressure; $\mathrm{A}-\mathrm{VO}_{2}$, arteriovenous oxygen difference; $\mathrm{VO}_{2}$, systemic oxygen consumption index. 


\section{EXERCISE TESTS}

Heart rate was monitored from a precordial lead. Arterial blood pressure was recorded from a cannula placed in the radial artery. A balloon tipped thermodilution catheter placed in the pulmonary artery was used to monitor pulmonary capillary wedge pressure and cardiac output (measured in duplicate using $10 \mathrm{ml}$ of iced saline). All pressures were measured in the upright posture with reference to the mid-chest. Arterial and mixed venous samples for gas analysis were drawn from the radial artery and the pulmonary artery respectively within one minute of the cardiac output measurements. Blood oxygen content, arteriovenous oxygen difference, systemic oxygen consumption index, and derived haemodynamic variables were calculated using standard formulas.

All patients were exercised on a treadmill. The Bruce protocol was used in eight patients and the less demanding Naughton protocol in two, both of whom had severely reduced exercise capacity. The same exercise protocol was used throughout the study in individual patients. Once maximal exercise capacity had been established in the preliminary test an arbitrary $75 \%$ submaximal level was calculated. In all subsequent exercise tests haemodynamic measurements were made at rest (resting 1), after two minutes of exercise, at submaximal exercise, and at maximal exercise. A final set of measurements was made during recovery (resting 2), one minute after maximal exercise. Blood samples for gas analysis were drawn at rest and at submaximal exercise. In individual patients the same level of submaximal exercise was used in each test to provide a directly comparable set of data. Values are expressed as mean (standard error of mean (SEM)).

\section{STATISTICAL ANALYSIS}

Analysis of variance was used to test the overall significance of the changes at each level of exercise during the course of the study. Those variables which

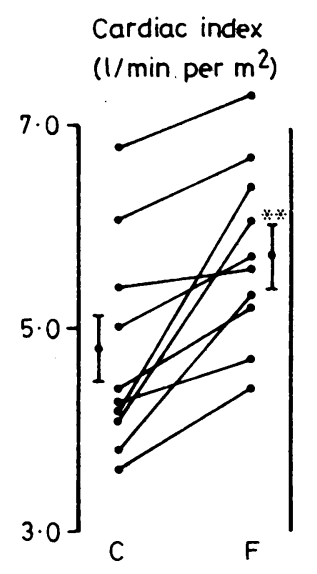

Systolic blood pressure $(\mathrm{mmHg})$

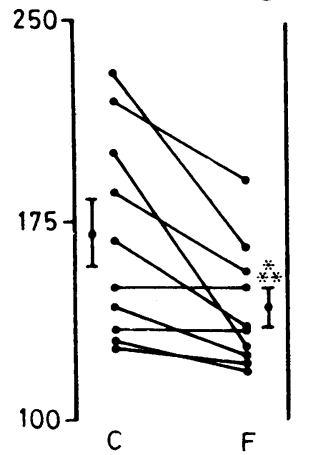

Stroke volume index $\left(\mathrm{ml} /\right.$ beat per $\mathrm{m}^{2}$ )

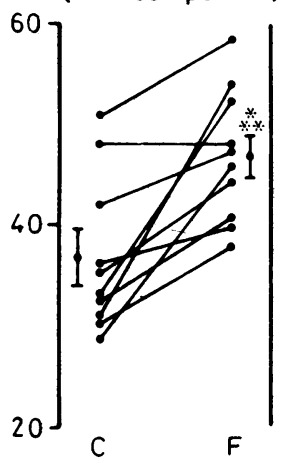

Systemic vascular resistance (units)

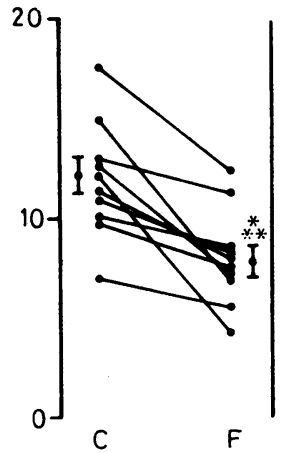

Pulmonary capillary wedge pressure $(\mathrm{mmHg})$ Heart rate (beats/min)

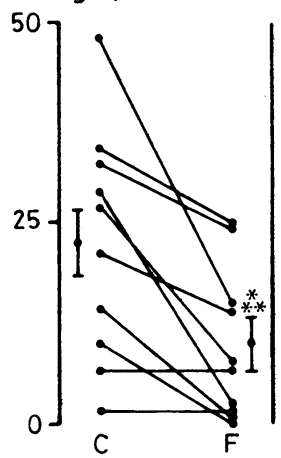

Arteriovenous oxygen difference (vol/100 $\mathrm{ml}$ )

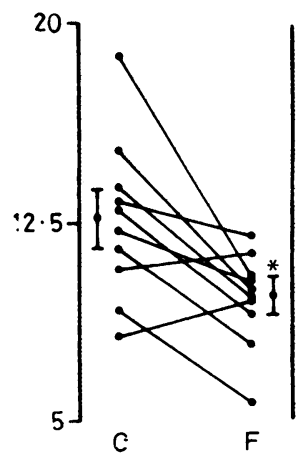



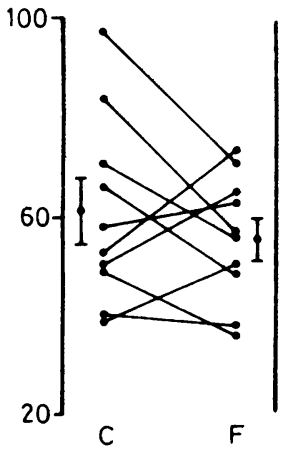

Fig 1. Effects of felodipine on haemodynamic variables and whole body oxygen utilisation at submaximal exercise. Individual values at control $(C)$ and after 28 days of felodipine $(F)$ are shown. Bars represent mean $(S E M)$ values. Significance $(p)$ of changes with respect to control: $\star<0.05, \star \star<0.01, \star \star \star<0.001$. 
Table 3 Duration of exercise to exhaustion and plasma felodipine concentrations in 10 patients with congestive heart failure. Values are mean (SEM)

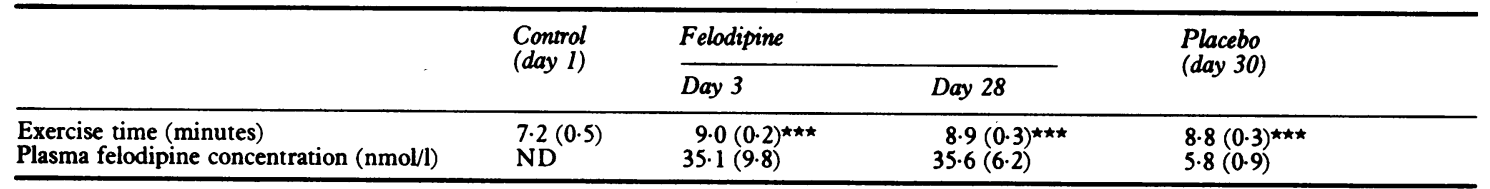

Significance (p) of changes in exercise time with respect to control: $\star \star \star<0.001$.

ND, not detectable.

changed significantly $(p<0.05)$ were then subjected to a modified analysis using the Bonferonni method to identify the source of the differences. 9

\section{Results}

Table 2 summarises the haemodynamic responses to treadmill exercise before, during, and after long term treatment with felodipine. Figure 1 shows the effects
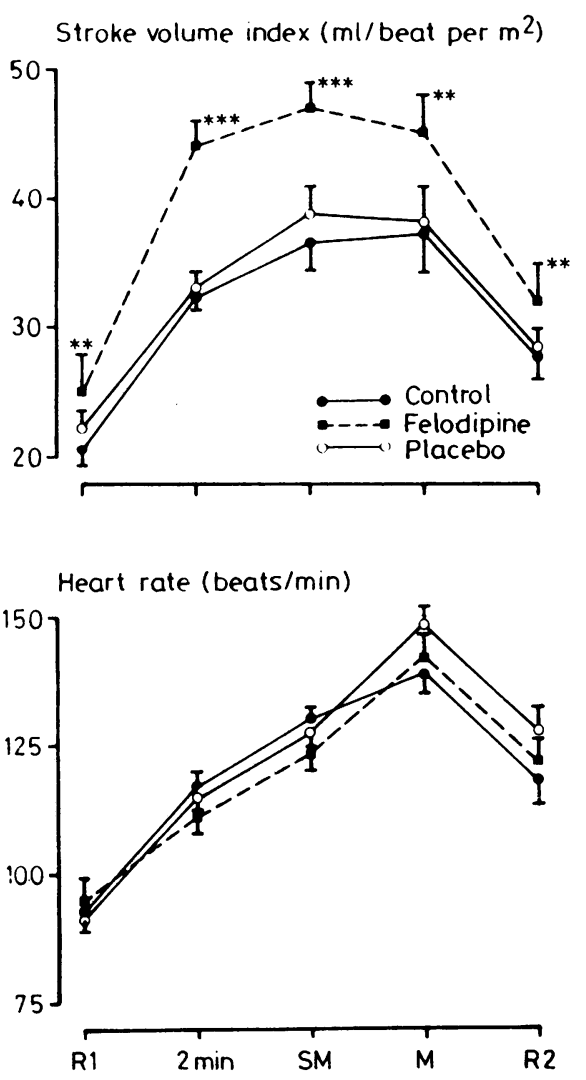

of felodipine on individual haemodynamic variables and on whole body oxygen utilisation at submaximal exercise.

\section{CONTROL DATA (DAY 1)}

Though the resting pulmonary capillary wedge pressure in the supine position was increased at $17(3) \mathrm{mm}$ $\mathrm{Hg}$ it fell to 9 (4) $\mathrm{mm} \mathrm{Hg}$ on standing. The resting erect values for cardiac index $\left(1.9(0.1) 1 / \mathrm{min} / \mathrm{per} \mathrm{m}^{2}\right)$

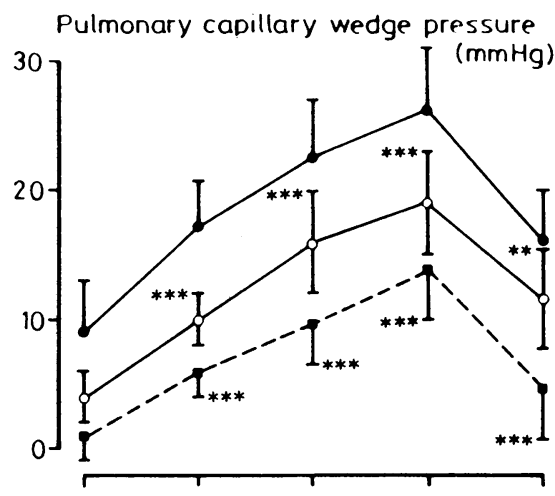

Systemic vascular resistance (units)

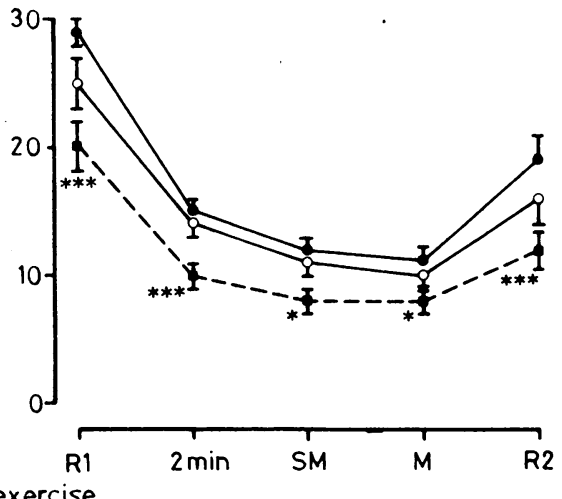

Fig. 2 Haemodynamic responses to exercise: mean values (SEM) at rest (RI), after two minutes (2 min) of treadmill exercise, at submaximal (SM) and maximal $(M)$ treadmill exercise, and during recovery $(R 2)$ one minute after maximal exercise. The felodipine data are for day 28 of the study. Significance $(p)$ of the felodipine and placebo effects with respect to control: $\star<0.05, \star \star<0.01$, $\star \star \star<0.001$. 
and stroke volume index (20(1) $\mathrm{ml} /$ beat $/$ per $\left.\mathrm{m}^{2}\right)$, however, both showed pronounced reductions below the normal range. The mean duration of exercise to exhaustion was $7 \cdot 2(0.5)$ minutes (Table 3). During exercise, pulmonary capillary wedge pressure rose steeply to $26(5) \mathrm{mm} \mathrm{Hg}$ at peak exercise, whereas cardiac index and stroke volume index rose to only 5.2 (0.5) $1 / \mathrm{min} /$ per $\mathrm{m}^{2}$ and $37(3) \mathrm{ml} /$ beat $/$ per $\mathrm{m}^{2}$ respectively (Fig. 2). Considerable increments in arteriovenous oxygen difference and systemic oxygen consumption index were also noted.

\section{FELODIPINE TREAT.MENT (DAYS 3 AND 28).}

Felodipine treatment produced a rapid improvement in both resting and exercise haemodynamics, which was sustained throughout the four week treatment period. The mean duration of exercise increased by $24 \%$ (Table 3 ). The pulmonary capillary wedge pressure was lower at rest and at every level of exercise and never exceeded $16(4) \mathrm{mm} \mathrm{Hg}$. Cardiac index and stroke volume index also improved considerably during this period rising to $6 \cdot 7(0.6) 1 / \mathrm{min} /$ per $\mathrm{m}^{2}$ and 48 (4) $\mathrm{ml} /$ beat/per $\mathrm{m}^{2}$ respectively at maximal exercise. Blood pressure at rest and during exercise and systemic vascular resistance were also significantly reduced but heart rate was unaffected by felodipine treatment (Fig. 2). These beneficial haemodynamic effects were associated with a pronounced decline in the arteriovenous oxygen difference, although systemic oxygen consumption index remained unchanged (Fig. 1). Plasma felodipine concentrations on days 3 and 28 of the study were similar (Table 3).

\section{PLACEBO (DAY 30)}

Two days after felodipine withdrawal plasma concentration had fallen to $5 \cdot 8(0.9) \mathrm{nmol} / \mathrm{l}$, and a pronounced deterioration in the rest and exercise haemodynamic variables was seen. Cardiac index and stroke volume index fell considerably and were not significantly different from the control values. Nevertheless, despite an increase in the pulmonary capillary wedge pressure above the treatment levels it remained significantly lower than control values during exercise (Fig. 2). The mean duration of exercise to exhaustion remained close to the level achieved during felodipine treatment (Table 3).

\section{DRUG RELATED SIDE EFFECTS}

Felodipine was generally well tolerated. Two patients reported some postural dizziness early during treatment. In both cases a small reduction in diuretic dosage was made, and the symptoms subsided within three days. A third patient developed mild noncardiac ankle oedema during treatment.

\section{Discussion}

These data show that felodipine treatment in congestive heart failure is well tolerated and improves the haemodynamic responses to treadmill exercise. The haemodynamic improvement is associated with an increase in exercise tolerance. Importantly, both effects are sustained during four weeks' treatment without evidence of tachyphylaxis.

Several short term clinical studies have shown that the vasodilator properties of calcium antagonists can improve left ventricular function in patients with congestive heart failure. ${ }^{10-12}$ Enthusiasm for this approach, however, has been tempered by reports of pulmonary oedema and circulatory collapse during treatment with these compounds ${ }^{13-15}$ due presumably to an adverse effect on myocardial contractility. ${ }^{1617}$ For this reason calcium antagonists are not at present widely used in heart failure. Nevertheless, the recent introduction of felodipine, a nifedipine analogue with pronounced vascular selectivity, promises to extend the clinical application of this class of agents. Experimental work has shown that felodipine is a potent vasodilator with negligible effects on the myocardium. ${ }^{56}$ Short term clinical studies confirm that the drug is without detectable negative inotropic properties. ${ }^{818}$ This distinguishes felodipine from other currently available calcium antagonists and suggests that the drug might be used safely in patients with congestive heart failure. The data we have presented support this suggestion.

The patients included in this study all had severe left ventricular dysfunction. Adequate diuretic treatment ensured that the resting pulmonary capillary wedge pressure in the upright posture was normal, although cardiac output was considerably reduced. The responses to treadmill exercise were typical for patients of this type.1920 An attenuated increase in cardiac output was seen despite a rapid and exaggerated rise in pulmonary capillary wedge pressure. Exercise capacity, therefore, was reduced because of the early onset of fatigue and dyspnoea.

The vasodilator properties of felodipine lowered blood pressure and systemic vascular resistance at rest and produced a substantial increase in cardiac output. Heart rate was unaffected indicating that the improvement in cardiac output was due entirely to an increase in stroke volume. The improvement in stroke volume will itself have tended to reduce the pulmonary capillary wedge pressure. ${ }^{21}$ Our data, however, do not exclude simultaneous drug induced venous pooling even though calcium antagonists usually have relatively little effect on the capacitance vessels. ${ }^{22}$ Similar beneficial effects were apparent during exercise. Thus at every level of exercise the pulmonary capillary wedge pressure was lower and the cardiac 
index was higher with felodipine. Consequently, exercise capacity increased considerably. That the haemodynamic improvement was a direct result of the drug intervention is confirmed by the fact that felodipine withdrawal led to a sharp reduction in plasma concentrations associated with a rapid deterioration in left ventricular function towards the baseline level.

Previous investigations of vasodilator treatment in congestive heart failure have shown a similar improvement in cardiac output at submaximal exercise, but reductions in left ventricular filling pressure usually occur only with drugs such as nitrates and prazosin, which have venodilator activity. ${ }^{23-25}$ Importantly, however, these studies have not always been able to show improvement in these haemodynamic variables at maximal exercise. ${ }^{26} 27$ In this respect, therefore, felodipine appears to have advantage over some of the currently available vasodilators since useful effects on both the filling pressure and the output of the left ventricle were seen both at submaximal and at maximal exercise.

In the present study a four week treatment period was used to evaluate the long term efficacy of felodipine. Although relatively short, this period was chosen in order to minimise the confounding influence of deteriorating left ventricular function on the data interpretation. The findings show that both the plasma concentrations and the beneficial haemodynamic effects of felodipine are sustained, and tolerance to the drug does not occur. This represents an additional advantage of felodipine since early tachyphylaxis has been reported with several other vasodilators used for the treatment of heart failure..$^{2-4}$ Prazosin, in particular, is subject to tolerance within the first several doses, ${ }^{34}$ although the clinical significance of this phenomenon remains uncertain and recent studies have been able to demonstrate long term efficacy. ${ }^{28} 29$

The increase in exercise cardiac output produced by felodipine was reflected by a significant reduction in the arteriovenous oxygen difference measured at submaximal exercise. Systemic oxygen consumption, however, was unaffected at this level of exercise suggesting that the regional perfusion of working skeletal muscle did not rise appreciably in response to treatment. It must be recognised that our data provide no information on oxygen consumption at the substantially higher level of peak exercise which was achieved during felodipine treatment. Despite this the findings bear some similarity to those reported with other vasodilators 232426 and indicate that the drug induced rise in cardiac output at submaximal exercise may be directed principally to the cutaneous and visceral circulations rather than to skeletal muscle. Flow redistribution of this type has been reported during treat- ment with glyceryl trinitrate in the exercising rat. ${ }^{30}$ The sensitivity of systemic oxygen consumption as a measure of regional skeletal muscle flow is uncertain, but it is conceivable that local metabolic factors produce maximal dilatation of muscular arterioles during exercise such that pharmacological intervention is relatively more active elsewhere in the circulation. Thus the principal benefit of felodipine treatment during exercise might relate to attenuation of the rapid rise in left ventricular filling pressure rather than to the increase in cardiac output. Indeed, this might be true of other vasodilators, and recent work has shown that long term treatment with hydralazine-a predominantly arteriolar dilator with little effect on venous tone-is no more effective than placebo in improving exercise tolerance in patients with heart failure. ${ }^{2}$

Further support for the primary importance of reduced left ventricular filling pressure as mediator of the beneficial effects of felodipine treatment is provided by the changes in maximal exercise tolerance seen in the present study. Thus the haemodynamic improvement that occurred in response to felodipine was reflected by a considerable increase in maximal exercise capacity, which was sustained throughout the treatment period. Of particular interest, however, was the finding that exercise capacity showed no significant reduction two days after felodipine withdrawal despite a pronounced haemodynamic deterioration. This might, of course, reflect an exercise training effect, ${ }^{31}$ but the haemodynamic deterioration was manifested principally by a reduction in stroke volume which was not significantly different from control values. Pulmonary capillary wedge pressure, on the other hand, remained appreciably lower than the control value, presumably as a result of residual felodipine activity in the circulation. Thus the sustained improvement in exercise capacity after felodipine withdrawal was likely to have been due, at least in part, to persisting reduction of the left ventricular filling pressure.

Previous investigation has shown beneficial haemodynamic and metabolic responses to acute felodipine treatment in patients with severe congestive heart failure. ${ }^{78}$ The present study has confirmed that similar effects are sustained during long term treatment and are associated with improvement in exercise tolerance. Felodipine has several advantages over currently available vasodilators and is potentially a useful agent for the long term management of congestive heart failure.

\section{References}

1 Zelis R, Flaim SF, Moskowitz RM, Nellis SH. How much can we expect from vasodilator therapy in congestive heart failure? Circulation 1979; 59: 1092-7. 
2 Franciosa JA, Weber KT, Levine TB, et al. Hydralazine in the long-term treatment of chronic heart failure: lack of difference from placebo. Am Heart $\mathcal{F}$ 1982; 104: 58794.

3 Packer M, Meller J, Gorlin R, Herman MV. Hemodynamic and clinical tachyphylaxis to prazosinmediated afterload reduction in severe chronic congestive heart failure. Circulation 1979; 59: 531-9.

4 Elkayam U, Lejemtel TH, Mathur M, et al. Marked early attenuation of hemodynamic effects of oral prazosin therapy in chronic congestive heart failure. Am $\mathcal{F}$ Cardiol 1979; 44: 540-5

5 Ljung B. Vascular versus myocardial selectivity in vitro of $\mathrm{H} 154 / 82$, a new dihydropyridine [Abstract]. Blood Vessels 1980; 17: 154.

6 Boström S-L, Ljung B, Mårdh S, Forsen S, Thulin E. Interaction of the antihypertensive drug felodipine with calmodulin. Nature 1981; 292: 777-8.

7 Tweddel AC, Johnsson G, Pringle TH, Murray RG, Hutton I. Systemic and coronary haemodynamic effects of felodipine in patients with coronary heart disease. Eur Heart f 1983; 4: 699-705.

8 Timmis AD, Campbell S, Monaghan MJ, Walker L, Jewitt DE. Acute haemodynamic and metabolic effects of felodipine in congestive heart failure. $\mathrm{Br} \mathrm{Heart} \mathcal{F}$ 1984; 51: 445-51.

9 Wallenstein S, Zucker CL, Fleiss JL. Some statistical methods useful in circulation research. Circ Res 1980; 47: $1-9$.

10 Polese A, Fiorentini C, Olivari MT, Guazzi M. Clinical use of a calcium antagonistic agent (nifedipine) in acute pulmonary edema. Am ₹ Med 1979; 66: 825-30.

11 Klugmann S, Salvi A, Camerini F. Haemodynamic effects of nifedipine in heart failure. $\mathrm{Br}$ Heart $\mathcal{F}$ 1980; 43: 440-6.

12 Ludbrook PA, Tiefenbrunn AJ, Reed FR, Sobel BE. Acute hemodynamic responses to sublingual nifedipine: dependence on left ventricular function. Circulation 1982; 65: 489-98.

13 Gillmer DJ, Kark P. Pulmonary oedema precipitated by nifedipine. Br Med f 1980; 280: 1420-1.

14 Brooks N, Catell M, Pidgeon J, Balcon R. Unpredictable response to nifedipine in severe heart failure. $\mathrm{Br}$ Med $\mathcal{F}$ 1980; $281: 1324$.

15 Robson RH, Vishwanath MC. Nifedipine and betablockade as a cause of cardiac failure. $\mathrm{Br}$ Med $\mathcal{F}$ 1982; 284: 104.

16 Fleckenstein A. Specific pharmacology of calcium in myocardium, cardiac pacemakers and vascular smooth muscle. Annu Rev Pharmacol Toxicol 1977; 17: 149-66.

17 Elkayam U, Weber L, Rose J, McKay C, Rahimtoola S. Nifedipine versus hydralazine in the treatment of severe heart failure: an evidence for a negative inotropic effect of nifedipine [Abstract]. Circulation 1983; 68: (part III): III-8.

18 Sheridan DJ, Culling W, Ruttley MST. Effects of felodipine on acute haemodynamics and left ventricular function in beta blocked patients with coronary artery disease [Abstract]. Br Heart $\mathcal{F}$ 1984; 51: 101.

19 Vatner SF. Response of the failing heart to severe exercise [Abstract]. Clin Res 1976; 24: 422A.

20 Rapaport E, Wong M, Ferguson RE, Bernstein P, Wiegand $\mathrm{BD}$. Right ventricular volumes in patients with and without heart failure. Circulation 1965; 31: 531-41.

21 Smith TW, Braunwald E. The management of heart failure. In: Braunwald E, ed. Heart disease. Philadelphia: W B Saunders, 1980: 509-70.

22 Braunwald E. Mechanism of action of calcium-channelblocking agents. $N$ Engl $\mathcal{F}$ Med 1982; 307: 1618-27.

23 Rubin SA, Chatterjee K, Parmley WW. Metabolic assessment of exercise in chronic heart failure patients treated with short-term vasodilators. Circulation 1980; 61: $543-8$.

24 Hindman MC, Slosky DA, Peter RH, Newman GE, Jones RH, Wallace AG. Rest and exercise hemodynamic effects of oral hydralazine in patients with coronary artery disease and left ventricular dysfunction. Circulation 1980; 61: 751-8.

25 Massie BM, Kramer B, Haughom F. Acute and longterm effects of vasodilator therapy on resting and exercise hemodynamics and exercise tolerance. Circulation 1981; 64: 1218-26.

26 Franciosa JA, Cohn JN. Immediate effects of hydralazine-isosorbide dinitrate combination on exercise capacity and exercise hemodynamics in patients with left ventricular failure. Circulation 1979; 59: 1085-91.

27 Franciosa JA, Cohn JN. Effect of isosorbide dinitrate on response to submaximal and maximal exercise in patients with congestive heart failure. Am $\mathcal{F}$ Cardiol 1979; 43: $1009-14$.

28 Colucci WS, Wynne J, Holman BL, Braunwald E. Long-term therapy of heart failure with prazosin: a randomized double-blind trial. Am f Cardiol 1980; 45: $337-44$.

29 Bertel O, Burkart F, Bühler FR. Sustained effectiveness of chronic prazosin therapy in severe chronic congestive heart failure. Am Heart $\mathcal{f}$ 1981; 101: 529-33.

30 Weitzel RL, Flaim SF, Zellis R. Effects of nitroglycerin infusion on the hemodynamic response to exercise in rats in heart failure [Abstract]. Clinical Research 1979; 27: 442A.

31 Froelicher VF Jr, Brammell H, Davis G, Noguera I, Stewart A, Lancaster MC. A comparison of the reproducibility and physiologic response to three maximal treadmill exercise protocols. Chest 1974; 65: 512-7. 